\title{
Spontaneous Coronary Artery Dissection Diagnosed by Coronary Computed Tomography Angiography
}

\author{
Romero $\mathrm{MJ}^{1 *}$, Carreño JM11, Picon $\mathrm{R}^{1}$, Molano FJ11, Romero $\mathrm{F}^{2}$ \\ 1Departamento de Cardiología, Hospital Universitario Valme, Spain \\ ${ }^{2}$ Departamento de Radiodiagnóstico, Hospital Universitario Valme, Spain
}

\section{Case Report \\ Volume 2 Issue 4}

Received Date: August 15, 2018

Published Date: September 10, 2018

*Corresponding author: Dr. María José Romero Reyes, Department of cardiology, Hospital de Valme, Avenida de Bellavista, Sevilla, Spain, 41014, Tel: 955015 102; Email: mjromrey@hotmail.com

\begin{abstract}
Spontaneous coronary artery dissection is a rare cause and, in many cases under suspected, and under diagnosed as an acute coronary syndrome. The incidence is higher in women, and younger patients without the traditional cardiovascular risk factors.

Given the lack of appropriate randomized clinical trials, doubts remain regarding the best diagnostic method and the best therapeutic option for these patients.

We describe the case of a 48-year-old woman who debuted as an acute coronary syndrome with persistent ST-segment elevation, for whose diagnosis a multimodal approach with imaging techniques was necessary.
\end{abstract}

Keywords: Spontaneous Coronary Artery Dissection; Acute Coronary Syndrome; Myocardial infarction with nonobstructive coronary arteries (MINOCA); Acute Myocardial Infarction; Chest pain; Multidetector Computed Tomography

Abbreviations: ECG: Electrocardiogram; DCA: Descending Coronary Artery; CCT: Coronary Computed Tomography; SCAD: Spontaneous Coronary Artery Dissection; MI: Myocardial Infarction; CX: Circumflex Artery.

\section{Case Report}

A 48 year-old-woman without cardiovascular risk factors, under warfarin treatment and suspecting the presence of a antiphospholipid syndrome after multiple embolic ischemic events, arrived to the emergency room with thoracic pain. The electrocardiogram (ECG) showed an elevation of the ST segment in DI-aVL. We performed an urgent coronary angiography which showed a significant lesion on the first diagonal ostium and a moderated lesion on the proximal anterior descending coronary artery (DCA) that was treated in a conservative way because the vessel keep maintaining a TIMI 3 flow (Figure 1A). The presence of a thin circumflex artery (CX) was remarkable. Therefore the presence of a coronary dissection at that level was suspected (Figure 1B).

Four days after the initial event, she presented a new case of thoracic pain with an elevation of the ST segment in derivations of the high lateral wall and TnT values raised to $940 \mathrm{ng} / \mathrm{dL}$. After the nitroglycerin intravenous infusion, the pain disappeared and the ECG was normalized. We decided to perform a coronary computed tomography (CCT) that showed a light reduction with a 


\section{Open Access Journal of Cardiology}

decrease of the contrast agent that indicated a mural hematoma on the proximal segment of the DCA (Figure 1C) and confirmed coronary spontaneous dissection of the CX (Figure 1D \& 1E). During that event the patient was under dual antiplatelet therapy, heparin, ACE inhibitors and beta blockers and a conservative strategy was finally carried out.

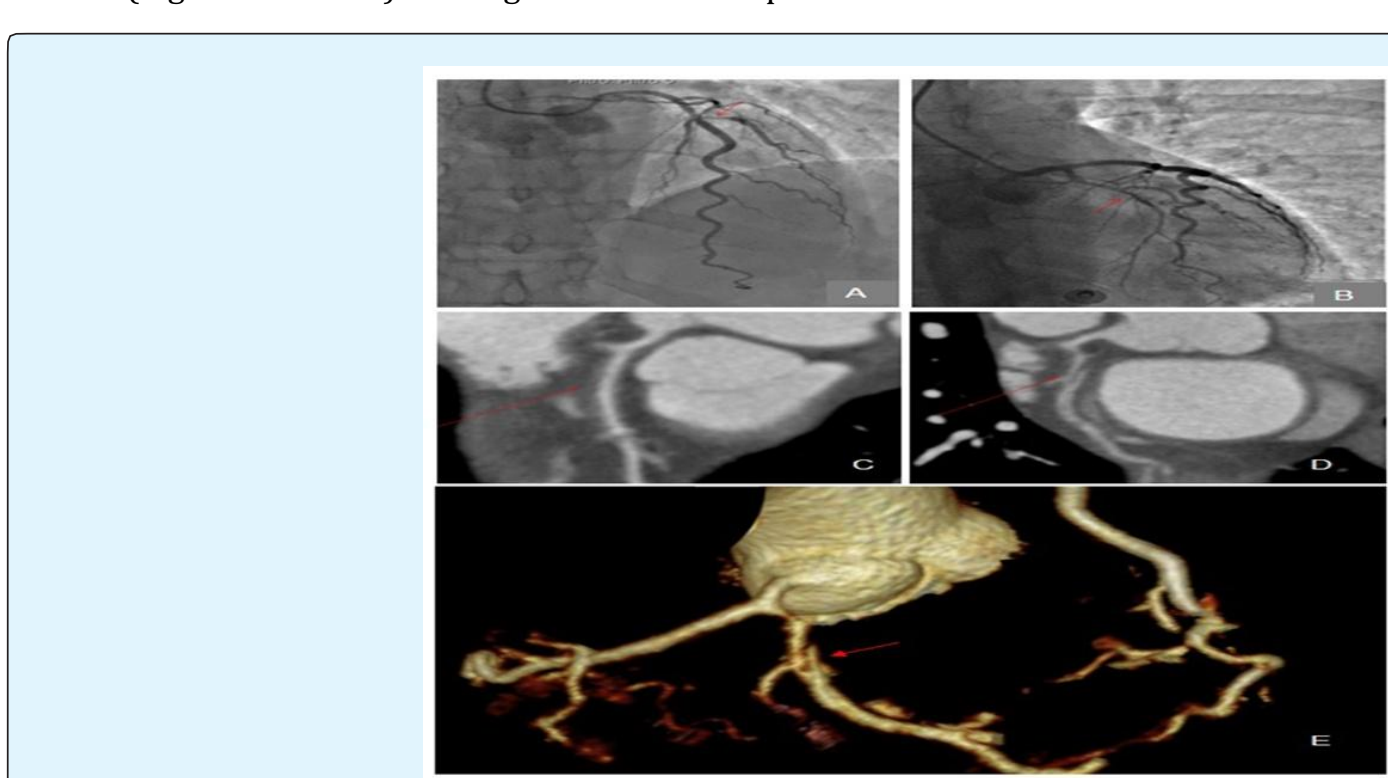

Figure 1: A) CCT showing moderate lesion in DCA and significant lesion in the first diagonal branch.

B) Invasive coronary angiography in which there is a decrease in the size of the CX.

C) CCT showing reduced light with contrast drop suggestive of mural hematoma in the proximal segment of the DCA.

D) CCT showing a linear hypointense image can be seen in the proximal CX, suggestive of coronary dissection. E) Volumetric 3D reconstruction of the CCT that shows the dissection in the proximal CX (arrow).

A cardiac resonance was performed for prognostic stratification that showed a subendocardial infarction of the mid-anteroseptal wall with microvascular obstruction of the lateral wall (Figure 2).
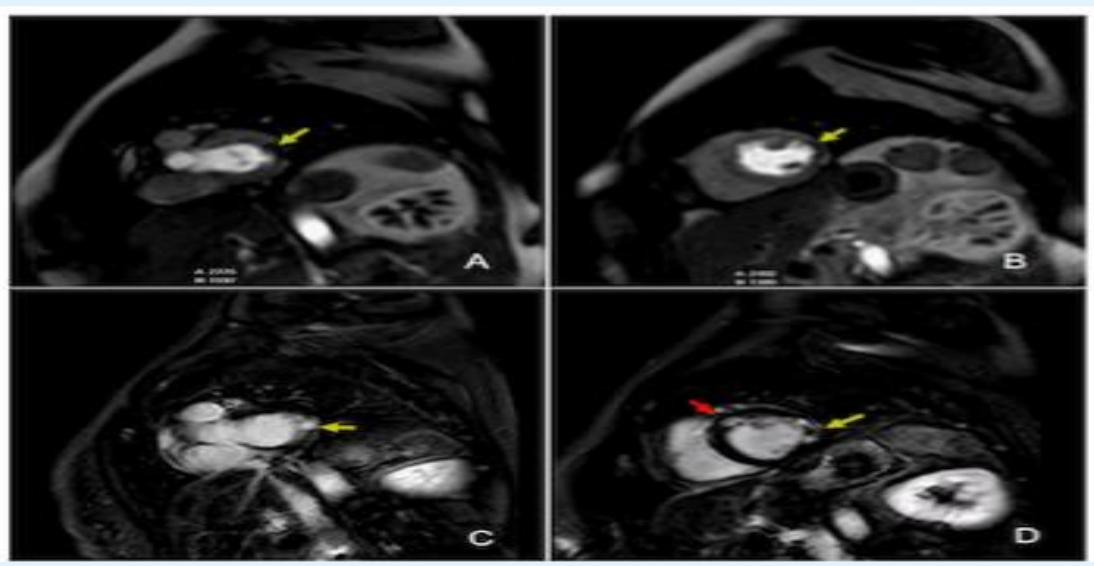

Figure 2: Cardioresonance study.

A \& B) First-pass perfusion sequence showing microvascular obstruction in the anterolateral and basal segments (arrow).

C \& D) Late gadolinium enhancement sequences showing transmural infarction with microvascular obstruction in the basal and middle anterolateral segments (arrow) and subendocardial necrosis in the middle anteroseptal segment (red arrow). 


\section{Open Access Journal of Cardiology}

After a favourable progress, the patient was discharged with triple antithrombotic therapy (warfarin, acetylsalicylic acid and clopidogrel) as well as ACEIs, highpotency statins and antianginal treatment and in the revision three months later included a stress echocardiography with dobutamine that showed hypokinesis of the basal and mid segments of the lateral wall without ischemia induction in the DCA.
A year after of the event, the patient presented a good functional preserved (LVEF of 60\%). Therefore, being the patient totally asymptomatic and suspecting a coronary artery dissection, a performed a control CCT was decided to avoid aggressive strategy, which showed certainly sealing spontaneous coronary artery dissection and disappearance of the intramural hematoma in the DCA and the first diagonal, without new lesions at any other level (Figure 3).
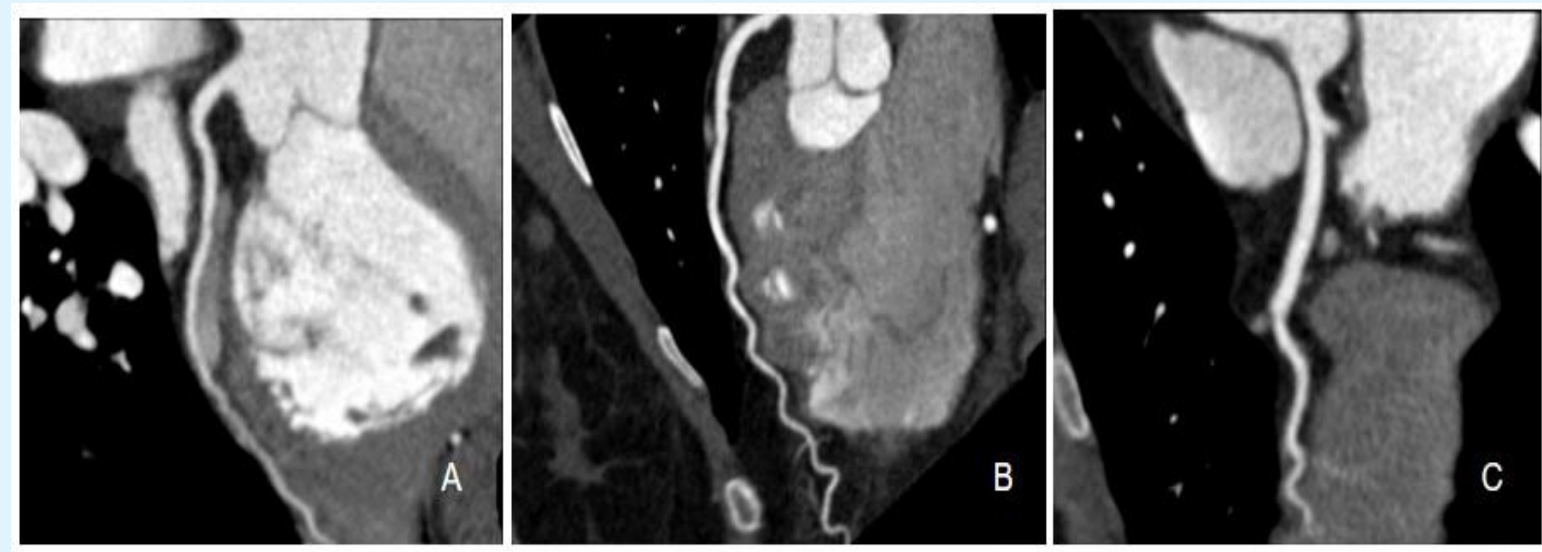

Figure 3: CCT performed a year after the event.

A) CX without lesion due to a spontaneous sealing of the dissection.

B \& C) DCA and first diagonal branch respectively without lesion.

\section{Discussion}

Spontaneous coronary artery dissection (SCAD) is a rare non-atherosclerotic cause of acute myocardial infarction (MI) and is frequently underdiagnosed. SCAD patients are often younger and more likely to be female without cardiovascular risk factors [1].

SCAD is caused by sudden separation of the inner intimal lining from the outer vessel wall and can be accompanied by bleeding from the vasa vasorum, resulting in intramural haematoma [1].

In most cases, an underlying disease is identified (up to $80 \%$ ), such as fibromuscular dysplasia, connective tissue diseases or postpartum [2]. In more than half of the cases, the SCAD occurs as non-ST-elevation MI (50-75\%), with less frequent ST-elevation myocardial infarction (25$50 \%$ ) [3]. DCA is the most frequent affected vessel (40$70 \%$ ) and single-vessel involvement is most common [4].

In most cases, the diagnosis of SCAD is made at the time of coronary angiography with the support of intracoronary diagnostic techniques such as intravascular coronary ultrasound or optical coherence tomography [4].

However, CCT is an alternative when invasive angiography is ambiguous or in the event that intracoronary diagnostic techniques have not been performed [5].

Conservative treatment is better for stable patients with SCAD [4]. Indications for revascularisation include recurrent ischemia, ongoing chest pain or haemodynamic instability and favorable anatomy, although it has a higher rate of complications compared to conservative treatment [5].

Although the survival rate described in both the short and long term is higher than in classic AMI, the rate of major cardiovascular events during the follow-up is high (estimated in $50 \%$ of cases at 10 years), including recurrence of the dissection (around 15\%), so it is necessary to perform close control on these patients [4]. 


\section{Open Access Journal of Cardiology}

\section{References}

1. Alfonso F (2012) Spontaneous coronary artery dissection: new insights from the tip of the iceberg? Circulation 126(6): 667-670.

2. Saw J, Ricci D, Starovoytov A, Fox R, Buller CE (2013) Spontaneous coronary artery dissection: prevalence of predisposing conditions including fibromuscular dysplasia in a tertiary center cohort. JACC Cardiovasc Interv 6(1): 44-52.

3. Saw J, Aymong E, Sedlak T, Buller CE, Starovoytov A, et al. (2014) Spontaneous coronary artery dissection: association with predisposing arteriopathies and precipitating stressors and cardiovascular outcomes. Circ Cardiovasc Interv 7(5): 645-655.

4. Saw J, Mancini GBJ, Humphries KH (2016) Contemporary Review on Spontaneous Coronary Artery Dissection. J Am Coll Cardiol 68(3): 297-312.

5. Manghat NE, Morgan-Hughes GJ, Roobottom CA (2005) Spontaneous coronary artery dissection: appearance and follow-up on multi-detector row CT coronary angiography. Clinical Radiology 60(10): 1120-1125. 